\title{
Heparin modification improves the re-endothelialization and angiogenesis of decellularized kidney scaffolds through antithrombosis and anti-inflammation in vivo
}

\author{
Jinbo Xie ${ }^{1 \#}$, Jian Wan ${ }^{2 \#}$, Xuemin Tang ${ }^{3}$, Wei Li ${ }^{1}$, Bo Peng ${ }^{1}$ \\ ${ }^{1}$ Department of Urology, Shanghai Tenth People's Hospital, School of Medicine, Tongji University, Shanghai, China; ${ }^{2}$ Center for Difficult and \\ Complicated Abdominal Surgery, Shanghai Tenth People's Hospital, School of Medicine, Tongji University, Shanghai, China; ${ }^{3}$ Department of \\ Nephrology, Wuhu No. 1 People's Hospital, Wuhu, China \\ Contributions: (I) Conception and design: J Xie, J Wan, W Li, B Peng; (II) Administrative support: J Wan, X Tang; (III) Provision of study materials \\ or patients: J Xie, J Wan; (IV) Collection and assembly of data: J Xie, J Wan; (V) Data analysis and interpretation: J Xie, X Tang; (VI) Manuscript \\ writing: All authors; (VII) Final approval of manuscript: All authors. \\ \#These authors contributed equally to this work. \\ Correspondence to: Wei Li; Bo Peng. Department of Urology, Shanghai Tenth People's Hospital, Tongji University School of Medicine, No. 301, \\ Yanchang Road, Jing'an District, Shanghai 200072, China. Email: liweitongji@163.com; pengbotgzy@163.com.
}

Background: Constructing tissue-engineered kidneys using decellularized kidney scaffolds (DKS) has attracted widespread attention as it is expected to be the key to solving the shortage of donor kidneys. However, thrombosis and the host inflammatory response are unfavorable factors that hider the reendothelialization and vascularization of the decellularized scaffolds.

Methods: Heparin was immobilized into the DKS using the method of 1-ethyl-3-(3-dimethylaminopropyl)carbodiimide/N-hydroxysuccinimide (EDC/NHS) activation. Fourier-transform infrared (FTIR) spectra were used to verify the heparinization of DKS. Human umbilical vein endothelial cells (HUVECs) were seeded and cultured in the DKS, then the sliced scaffolds were transplanted subcutaneously into nude mouse. Scanning electron microscopy and a series of histochemical stains including hematoxylin and eosin (H\&E), elastic Verhöeff-Van Gieson (EVG), Sirius red, Masson's trichrome, and toluidine blue (TB) staining were used for morphological characterization. The qRT-PCR analysis, immunohistochemistry (IHC), and immunofluorescence (IF) staining were used to determine the expression of related molecular markers.

Results: The rat DKS completely retained the extracellular matrix and heparinized modification. The H\&E staining results showed there were more HUVECs covering the internal surfaces of tubular structures in the HEP-DKS group compared with the DKS group. The IF analysis results revealed that CD31, Ki67, and CD206 had higher positive rates in HUVECs in the HEP-DKS group compared to the DKS group. Both groups of scaffolds showed blood vessel formation via $\mathrm{H} \& \mathrm{E}$ staining, and there were more blood vessels in the HEP-DKS group compared with the native DKS group $(\mathrm{P}<0.05)$. The qRT-PCR results showed that the levels of IL-1 $\beta$, IL-6, and TNF- $\alpha$ in the HEP-DKS group were significantly lower than those of the native DKS group, while the expression level of IL-10 was significantly higher than that in the native DKS group $(\mathrm{P}<0.05)$.

Conclusions: Heparin modification improves the re-endothelialization and vascular regeneration of the DKS through anticoagulation in vitro and in vivo. The anti-inflammatory effect of heparin on the transplanted host was initially confirmed, and it is considered that this effect may play a non-negligible role in promoting DKS re-endothelialization and angiogenesis. Heparinized DKS is therefore a promising candidate for kidney tissue engineering.

Keywords: Decellularized kidney scaffolds (DKS); heparin; endothelialization; angiogenesis; anti-inflammation

Submitted Jul 23, 2021. Accepted for publication Sep 16, 2021.

doi: $10.21037 /$ tau-21-703

View this article at: https://dx.doi.org/10.21037/tau-21-703 


\section{Introduction}

Compared with blood purification, kidney transplantation is recognized as a more effective treatment for end-stage renal disease (ESRD) in terms of long-term survival and quality of life. However, the serious imbalance between the supply and demand of donor organs has become the main bottleneck restricting the widespread implementation of kidney transplantation (1). The development of regenerative medicine and tissue engineering has brought about promising new strategies for solving the shortage of kidney transplant donors during the past few decades, one of which is the development of decellularized kidney scaffolds (DKS) (2).

Decellularized scaffolds have been studied in various organs, including the heart, liver, spleen, kidney, and pancreas. Like the decellularized scaffolds of other organs, the most prominent advantage of DKS is that the natural tissue extracellular matrices (ECMs) retain the microstructure and many irreproducible bioactive components while removing multiple antigen components maximally, which can help cell adhesion and regeneration without being rejected by the transplant recipient (2). Researchers have attempted to generate decellularized scaffolds using the kidneys of various mammals, including porcine, rat, monkey and so on (1-3), but regardless of the animal the DKS is derived from, in order to be transplanted into the body, the organ must generate enough unblocked blood vessels through seeding cells. How to achieve reendothelialization and reduce vascular thrombosis and the inflammatory stress of transplant recipients within a reasonable length of time are common burning issues faced by various decellularized scaffolds towards practical applications. To address these problems, researchers have tried to optimize the decellularized scaffolds though various modification methods, including immobilization of heparin (4,5), sugar (6), GRGDSPC peptides (7), anti-endothelial cell (EC) antibodies (8), and stromal cell-derived factor-1 alpha loaded proteolytically degradable hydrogel (9).

Recently, heparin has received increased attention for modifying decellularized scaffolds. Heparin is widely used clinically because of its anticoagulant, anti-inflammatory, and other pharmacological properties. The study of heparin modification in DKS is in its infancy, and many functional effects are still unclear. Wang et al. (4) verified the antiplatelet adhesion effect of heparin by perfusing wholeblood into DKS and the promoting recellularization effect of heparin via whole-organ culture of cell-seeded DKS in vitro. However, the effect of heparin modification on the reendothelialization of DKS has not been explored in vivo, and the immune environment of DKS transplantation host is also an important factor affecting histocompatibility. In the present study, heparin was immobilized into DKS then activated using 1-ethyl-3-(3-dimethylaminopropyl)carbodiimide/N-hydroxysuccinimide (EDC/NHS). Then, the DKS was characterized using histochemical and composition analysis methods, and the recellularization, angiogenesis, and immune status were assessed in vitro or in vivo (Figure 1). We present the following article in accordance with the ARRIVE reporting checklist (available at https://dx.doi.org/10.21037/tau-21-703).

\section{Methods}

\section{Animals and cells}

Mature male Sprague Dawley (SD) rats and Balb/c nude mice were purchased from the Laboratory Animal Research Center, Tongji University. The animals were kept under specific pathogen-free (SPF) conditions, maintained on a 12-h light/dark cycle, and were given free access to standard laboratory feed and water. All animal work was approved by the Animal Ethics Committee of Shanghai Tenth People's Hospital, in compliance with national guidelines for the care and use of animals. A protocol was prepared before the study without registration. Human umbilical vein endothelial cells (HUVECs) were obtained from the Type Culture Collection of the Chinese Academy of Sciences (Shanghai, China) and cultured in EC medium (ScienCell, USA).

\section{Harvest and decellularization of rat kidneys}

Kidneys were harvested from male SD rats weighing $300 \pm 20$ g. Briefly, anesthetized rats were systemically anticoagulated with heparin, and $22 \mathrm{G}$ cannulae were inserted into the renal artery and ureter and fixed with 4-0 Mersilk. Firstly, the kidney was arterially perfused in situ using phosphate buffered saline (PBS) to remove the blood. Then, the artery cannula was connected into a peristaltic pump (Masterflex, USA) to permit the flow of the perfusate, and the kidney was perfused with $1 \%$ Triton $\mathrm{X}-100 / 0.1 \%$ ammonium hydroxide (Sigma-Aldrich, USA) for $12 \mathrm{~h}$ at $1 \mathrm{~mL} / \mathrm{min}$. Finally, the kidney was perfused with PBS containing $100 \mathrm{U} / \mathrm{mL}$ penicillin $/ 100 \mu \mathrm{g} / \mathrm{mL}$ streptomycin (Sigma-Aldrich, USA) and $0.0025 \%$ deoxyribonuclease 1 (DNase; Sigma-Aldrich, USA) for $24 \mathrm{~h}$ to clear the remaining cellular and DNA debris. 

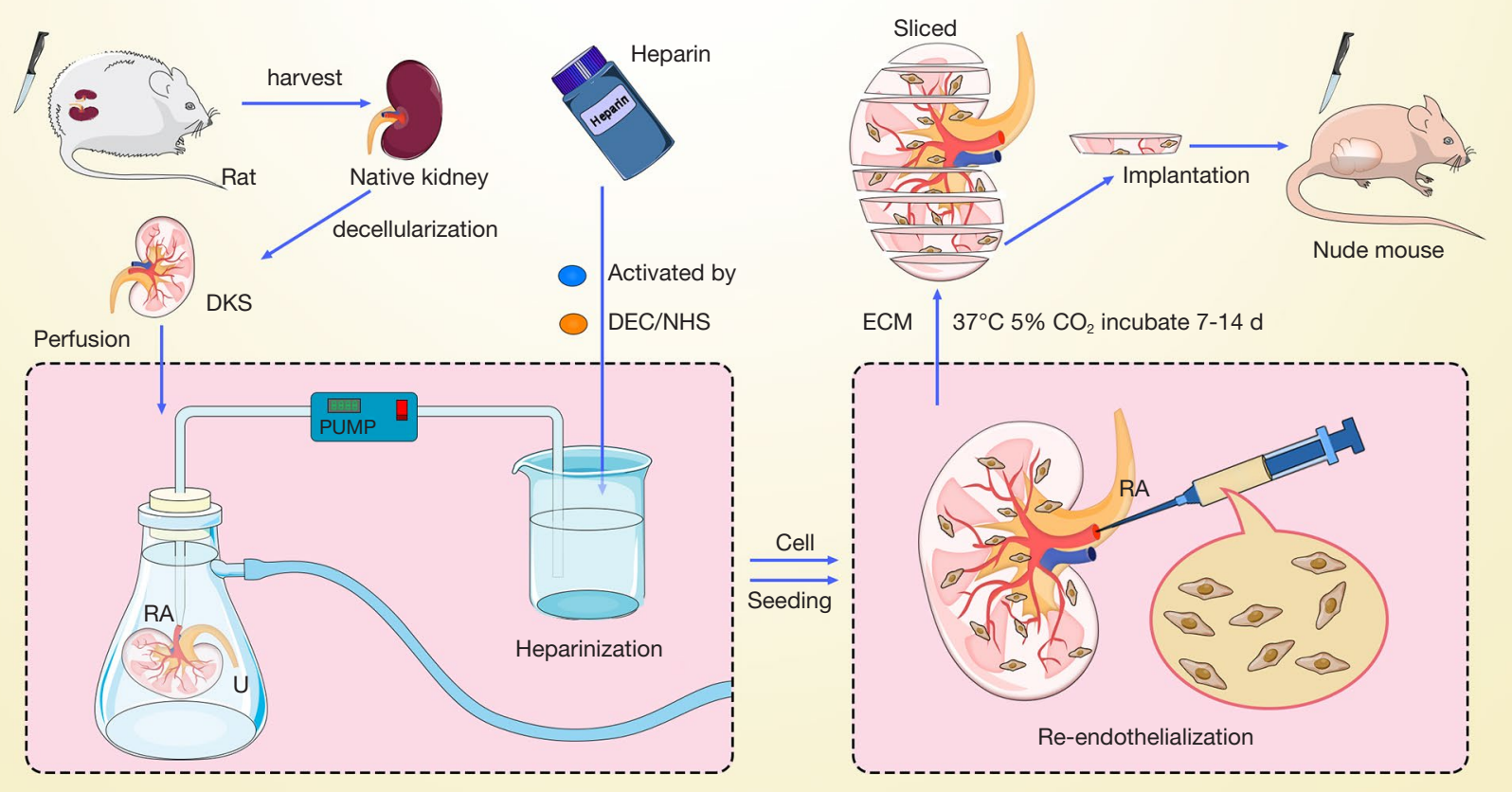

Figure 1 Schematic of the research protocol. DKS, decellularized kidney scaffold; ECM, extracellular matrix; EDC/NHS, 1-ethyl-3-(3dimethylaminopropyl)-carbodiimide/N-hydroxysuccinimide.

Scanning electron microscope was used to verify the complete $3 \mathrm{D}$ microstructure of DKS.

\section{Heparin modification of DKS}

Heparin was immobilized into DKS using the method described in a previous study (10). Firstly, heparin sodium (H-4784; Sigma-aldrich, USA), with a concentration of $1 \mathrm{mmol} / \mathrm{L}$ and average molecular weight of $15 \mathrm{kDa}$, was dissolved in morpholine ethanesulfonic acid buffer with $\mathrm{pH}$ 6.5. Secondly, $120 \mathrm{mmol} / \mathrm{L}$ EDC and $60 \mathrm{mmol} / \mathrm{L}$ NHS were added into and activated the heparin solution for $4 \mathrm{~h}$ at room temperature. Then, the DKS was perfused for $4 \mathrm{~h}$ at $37^{\circ} \mathrm{C}$ with the activated heparin solution via the perfusion system. Subsequently, the DKS was sequentially washed with $\mathrm{Na} 2 \mathrm{HPO} 4$ for $2 \mathrm{~h}(0.1 \mathrm{M}), \mathrm{NaCl} 4$ times in $24 \mathrm{~h}(4 \mathrm{M})$, and double distilled water 5 times in $24 \mathrm{~h}$.

\section{Characterization of heparin modification}

The heparinized DKS (HEP-DKS) and the native DKS were fixed in $4 \%$ paraformaldehyde overnight at $4{ }^{\circ} \mathrm{C}$. After washing with running water, both were embedded in paraffin, and the tissue was sliced continuously with a thickness of
$5 \mu \mathrm{m}$. After drying the sections at $60^{\circ} \mathrm{C}$ and with xylene, sections were dewaxed with xylene, dehydrated with gradient ethanol, and then dyed with a newly prepared $1 \%$ toluidine blue (TB) solution for $30 \mathrm{~min}$. After washing off the dye solution, sections were dehydrated in gradient ethanol and xylene until transparent. Finally, the slides were mounted with neutral resin and images were captured under an upright microscope. Fourier-transform infrared (FTIR) spectra (Nicolet 560, USA) were used to detect the heparin sodium immobilized into the DKS as per the operating procedure.

\section{Recellularization of DKS}

HUVECs were cultured in EC medium with $5 \%$ fetal bovine serum (FBS), 1\% EC growth supplement, and 1\% penicillin/streptomycin solution. For re-endothelialization of the scaffolds, $2.5 \times 10^{5} / \mathrm{mL}$ HUVEC suspensions were injected into native DKS and HEP-DKS through the renal artery, and the injection was repeated 2-3 times throughout the recellularization experiment. Subsequently, the cellinfused scaffolds were put in an incubator at $37^{\circ} \mathrm{C}$ and $5 \%$ $\mathrm{CO}_{2}$ for culture, the EC medium was renewed regularly, and the scaffolds were harvested for further experiments after $7-14$ days. 
Table 1 The primer sequences used in this study

\begin{tabular}{ll}
\hline Primer & Sequence (5' to ${ }^{\prime}$ ') \\
\hline GAPDH-F & AAGAAGGTGGTGAAGCAGG \\
GAPDH-R & GAAGGTGGAAGAGTGGGAGT \\
IL-1 $\beta-F$ & GAAATGCCACCTTTTGACAGTG \\
IF-1 $\beta-R$ & TGGATGCTCTCATCAGGACAG \\
IL-6-F & CTGCAAGAGACTTCCATCCAG \\
IL-6-R & AGTGGTATAGACAGGTCTGTTGG \\
IL-10-F & CTTACTGACTGGCATGAGGATCA \\
IL-10-R & GCAGCTCTAGGAGCATGTGG \\
TNF- $\alpha-F$ & CTGAACTTCGGGGTGATCGG \\
TNF- $\alpha-R$ & GGCTTGTCACTCGAATTTGAGA \\
\hline
\end{tabular}

\section{Hematoxylin and eosin (H\&E), elastic Verhöeff-Van Gieson (EVG), Sirius red, and Masson's trichrome staining}

The native rat kidney and HEP-DKS were fixed in 4\% paraformaldehyde overnight at $4{ }^{\circ} \mathrm{C}$. After rinsing with running water, they were embedded in paraffin, and the tissue was sliced continuously with a thickness of $5 \mu \mathrm{m}$. For H\&E staining, the sections were dried at $60{ }^{\circ} \mathrm{C}$, deparaffinized with xylene, dehydrated with gradient ethanol, and stained with eosin and hematoxylin respectively. Finally, the slides were sealed with neutral gum, and then imaged with an optical microscope. For EVG staining, the sections were dyed in Verhöeff's solution and redyed with Van Gieson's solution. For Sirius red staining, the sections were dyed in $0.1 \%$ Sirius red solution. For Masson's trichrome staining, the sections were successively dyed in Mayer's hematoxylin, acid ponceau, and aniline blue or brilliant green. In the above various dyeing methods, except for the special dyes used, the other steps were similar.

\section{Immunobistochemistry (IHC) and immunofluorescence (IF) analysis}

The tissues were prepared for IHC and IF staining according to the protocol for paraffin embedding. For IHC staining, the native rat kidney sections were incubated with primary antibodies including anti-collagen I 1:100 (ab270993, Abcam, UK), anti-collagen IV 1:100 (ab6586, Abcam, UK), and anti-fibronectin 1:100 (ab2413, Abcam, UK) at $4{ }^{\circ} \mathrm{C}$ overnight. Then, the sections were incubated with secondary biotinylated goat anti-rabbit antibodies (Solarbio, China). For IF staining, the sections of HEP-DKS and native DKS were incubated with primary antibodies including anti-CD31 (\#3528, CST, USA), anti-Ki67 (\#9449, CST, USA), and anti-CD206 (\#24595, CST, USA) at $4{ }^{\circ} \mathrm{C}$ overnight. Then, the sections were incubated with secondary Alexa Fluor 488-conjugated goat anti-mouse/antirabbit antibodies 1:500 (Thermo Fisher Scientific, USA) for $1 \mathrm{~h}$. Subsequently, the sections were stained with DAPI for $10 \mathrm{~min}$. All the above sections were observed and imaged using an upright microscope (Leica, Germany).

\section{In vivo implantation study}

The native DKS and HEP-DKS with re-endothelialization were sectioned into $5 \mathrm{~mm}$ thick slices for subcutaneous implantation. Balb/c mice ( $\mathrm{n}=5)$ were anesthetized using sodium pentobarbital solution, and one $1.5 \mathrm{~cm}$ incision was made in the dorsal surface where the tissue slices were implanted through suturing. The samples were harvested and analyzed using H\&E staining and IF staining.

\section{qRT-PCR analysis}

Total RNA was extracted using the Trizol kit (TakaRa, Japan). In the present study, the HEP-DKS and native DKS were sliced and transplanted subcutaneously into nude mice, and were harvested after 3 weeks. Then the total RNA of harvested HEP-DKS and native DKS were respectively extracted using the Trizol kit. The qRT-PCR was performed using the RT reagent kit (TakaRa, Japan) following the protocol for the ABI Prism 7500 sequence detection system (Applied Biosystems, USA). The results were normalized using $\beta$-actin mRNA. The expression levels were analyzed using the $2^{-\Delta \Delta \mathrm{Ct}}$ method. The primer sequences used in this study are listed in Table 1.

\section{Statistical analysis}

Graphpad Prism 8 was used for statistical analysis and graphing in this study. Student's $t$-test was used to analyze normally distributed data, and the significance level was set as $\mathrm{P}<0.05$.

\section{Results}

\section{Preparation and characterization of the DKS}

Isolated rat kidneys were decellularized via renal artery perfusion with $10 \%$ Triton $\mathrm{X}-100 / 0.1 \%$ ammonium hydroxide solution at a constant pressure of $30 \mathrm{mmHg}$ 


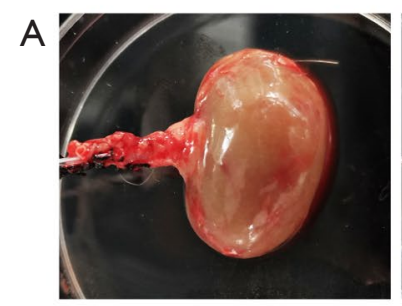

$\mathrm{Oh}$

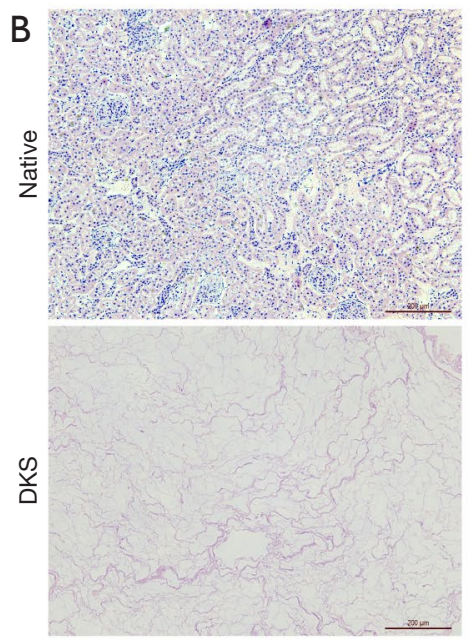

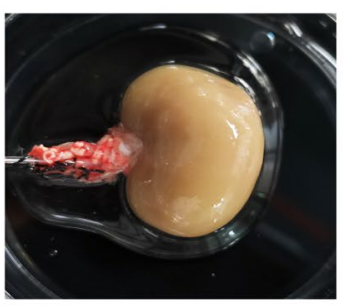

$4 \mathrm{~h}$

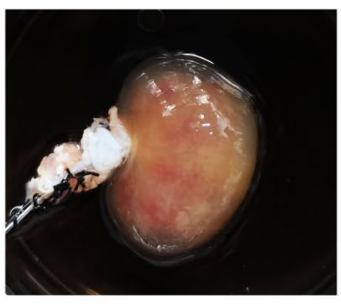

$8 \mathrm{~h}$

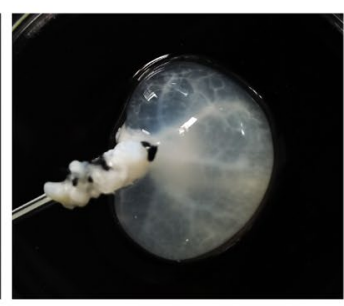

$12 \mathrm{~h}$
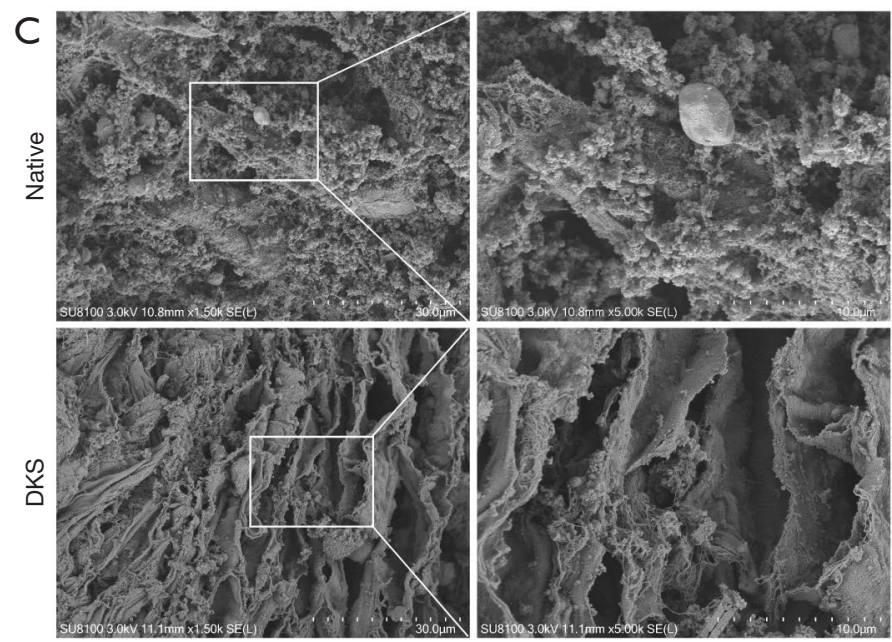

Figure 2 Arterial perfusion decellularization of rat kidneys and microstructure images. (A) Time lapse images of a freshly isolated rat kidney undergoing continuous low-flow renal arterial perfusion with 1\% Triton X-100/0.1\% ammonium hydroxide. (B) H\&E staining showed the absence of cells and the integrity of tubular structures in the DKS compared with the native kidney (200×). (C) SEM images of the DKS exhibited no residual cells and well-preserved 3D tubular architecture compared with the native organ. SEM, scanning electron microscopy; $\mathrm{H} \& \mathrm{E}$, hematoxylin and eosin.

(Figure $2 A$, time lapse images at $0,4,8$, and $12 \mathrm{~h}$ ). $\mathrm{H} \& \mathrm{E}$ staining images showed the absence of cells in the DKS compared with the native kidney (Figure $2 B$ ). The scanning electron microscopyimages indicated that the microstructure was not destroyed in the DKS (Figure 2C).

To further determine the integrity of the remaining ECM, a series of staining analyses were carried out, including EVG, Sirius red, and Masson's trichrome staining. The results showed that most of the collagen fiber components were preserved (Figure $3 A$ ). Moreover, the results of IHC indicated that collagen I, collagen IV, and fibronectin in the ECM were well retained (Figure 3B).

\section{Characterization of the DKS loaded with heparin}

TB staining was carried out to detect the heparinization of DKS, and the results indicated that the HEP-DKS group had significant positive TB staining, while there was no obvious color change in the control group (Figure 4A). Figure $4 B$ shows the FTIR spectra of the DKS with or without heparinization and pure heparin. The signals at approximately 1,160 and $1,747 \mathrm{~cm}^{-1}$ in the spectra of the HEP-DKS showed the characteristic signals of heparin, which verified that heparin was immobilized into the ECM of DKS.

\section{Re-endothelialization of the DKS and functional characterization in vitro}

After HUVECs were seeded into the native and heparinmodified DKS and cultured for 7 days, the scaffolds were harvested and further analyzed. H\&E staining was performed and the results showed that HUVECs adhered to both of the scaffolds. Moreover, there were more cells covering the internal surfaces of tubular structures in 


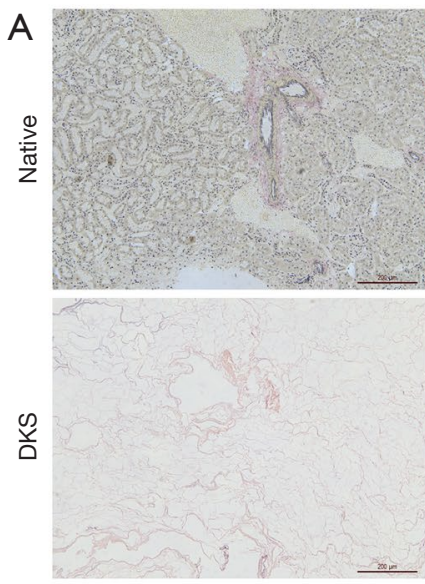

EVG

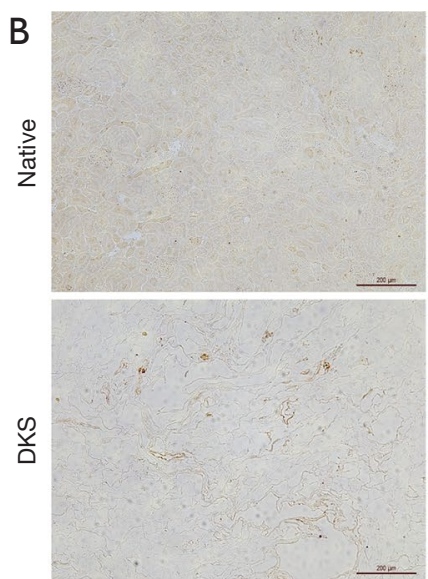

Collagen I

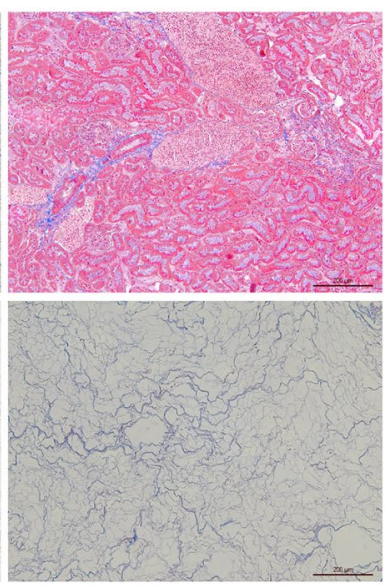

Masson's trichrome

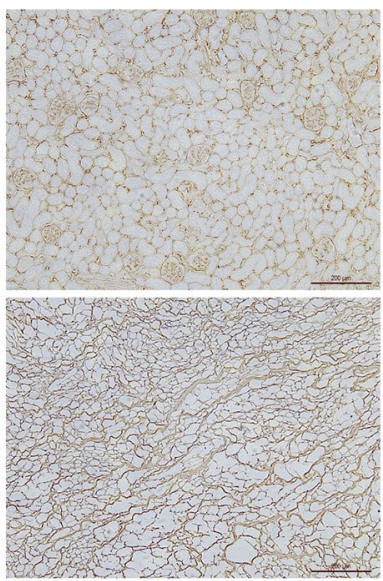

Collagen IV

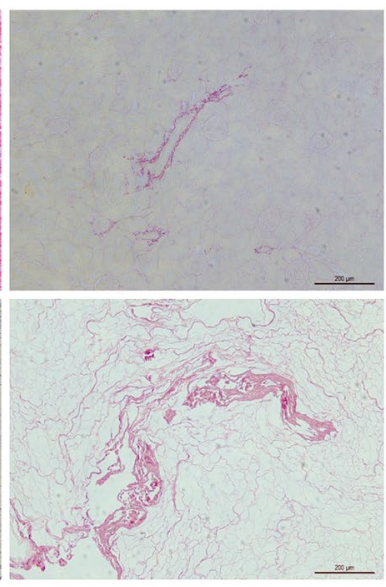

Sirius red

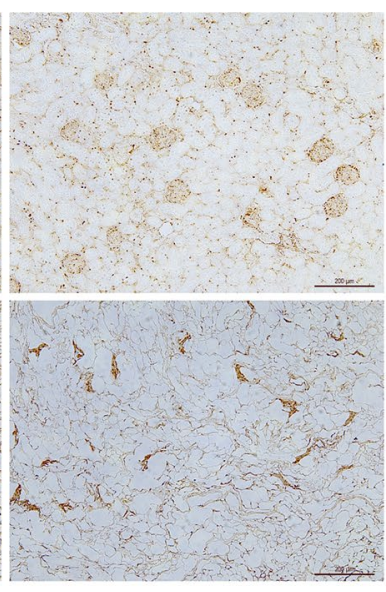

Fibronectin

Figure 3 ECM characterization of the rat DKS. (A) EVG staining, Masson's trichrome staining, and Sirius red staining showed most of the collagen fiber components were retained in the DKS compared with the native kidney (200x). (B) IHC of the native kidney and DKS: collagen I (left), collagen IV (middle), and fibronectin (right) (200x). ECM, extracellular matrix; DKS, decellularized kidney scaffold; EVG, Elastic Verhoeff-Van Gieson; IHC, immunohistochemistry.

the HEP-DKS group compared with the DKS group (Figure $5 \mathrm{~A}$ ). To assess the angiogenesis and proliferation capacities, IF analyses were conducted, and the results revealed that $\mathrm{CD} 31$ and $\mathrm{Ki} 67$ had a higher positive rate in HUVECs in the HEP-DKS group than in the DKS group (Figure 5B,5C).

\section{Angiogenesis and bost inflammatory response towards scaffolds in vivo}

The HEP-DKS and native DKS were sliced and transplanted subcutaneously into nude mice, and were harvested after 3 weeks. The gross appearances of implants indicated that both of the re-endothelialized scaffolds showed good histocompatibility with the subcutaneous tissue of nude mice (Figure $6 \mathrm{~A}$ left). Both groups of scaffolds showed blood vessel formation via $\mathrm{H} \& \mathrm{E}$ staining, and there were more blood vessels in the HEP-DKS compared with the native DKS (Figure $6 \mathrm{~A}$ middle and right). Angiogenesis ability was also evaluated via IF in vivo, and CD31 showed a higher positive rate in the cells of the HEP-DKS group compared with the DKS group (Figure 6B). In addition, the expression level of CD206 as the characteristic marker of M2 type macrophages was determined in both groups of scaffolds. The positive rate of CD206 in the cells of the HEP-DKS group was significantly higher than in the DKS 

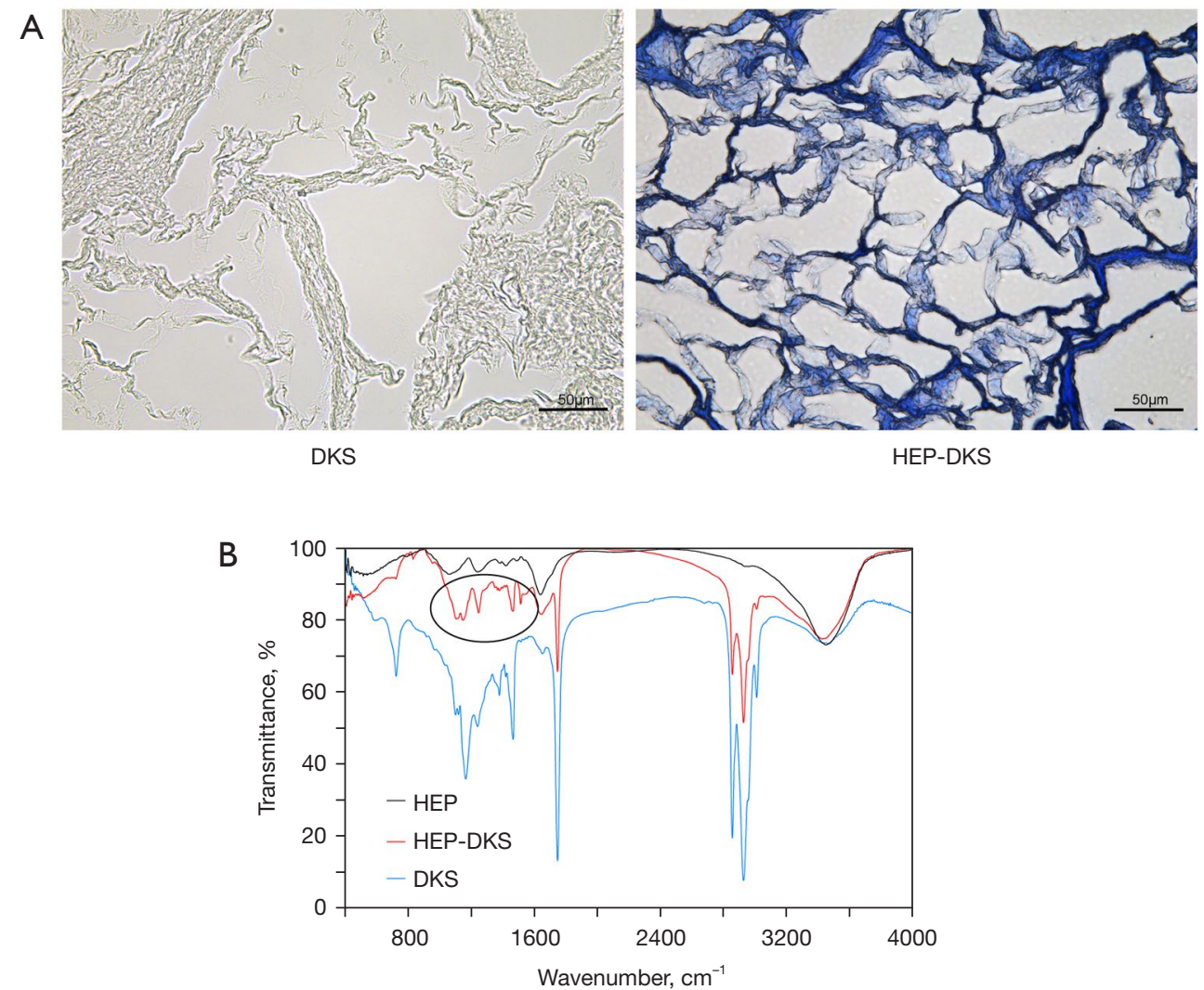

Figure 4 The characterization of the heparin immobilized DKS. (A) Histological toluidine blue staining of the native DKS and HEP-DKS. Blue staining was positive only in the HEP-DKS (400×). (B) FTIR spectra of heparin, HEP-DKS, and DKS. DKS, decellularized kidney scaffold; HEP-DKS, heparinized decellularized kidney scaffold; FTIR, Fourier-transform infrared.

group (Figure 6C).

In order to further evaluate the inflammatory responses of the transplanted host, we extracted the total RNA from the 2 groups of nude mice with subcutaneously implanted scaffolds and performed qRT-PCR analysis. The qRT-PCR results showed that the levels of IL- $1 \beta$, IL- 6 , and TNF- $\alpha$ in the HEP-DKS group were significantly lower than those in the native DKS group (Figure $7 A-7 C$ ), while the expression level of IL-10 was significantly higher than that in the native DKS group (Figure $7 D$ ).

\section{Discussion}

It is estimated that chronic kidney disease (CKD) may become the fifth leading cause of death worldwide by 2040 (11). Kidney transplantation, as one of the most promising methods to reverse this projection, is limited by a shortage of donors. The ultimate goal of renal tissue engineering is to produce a transplantable substitute with equivalent renal function for clinical application. The DKS has unique advantages because of its access to the maximum retention of the ECM microstructure and the minimum residual immunogenicity $(12,13)$. The growth factors and bioactive components contained in the ECM of DKS facilitate cell adhesion and can induce cell migration and differentiation (14). Appropriate decellularization methods can make the ECM suffer the slightest damage and retain its natural components and morphology to the greatest extent. In this study, method of $1 \%$ Triton $\mathrm{X}-100 / 0.1 \%$ ammonium hydroxide at $1 \mathrm{~mL} / \mathrm{min}$ was used to decellularize, obtaining DKS with a transparent appearance and complete vascularity, which reduced the damage of high-strength decellularization reagents, water impact and high hydrostatic pressure to the microstructure of ECM, especially the nephron.

Unfortunately, a series of challenges have gradually 

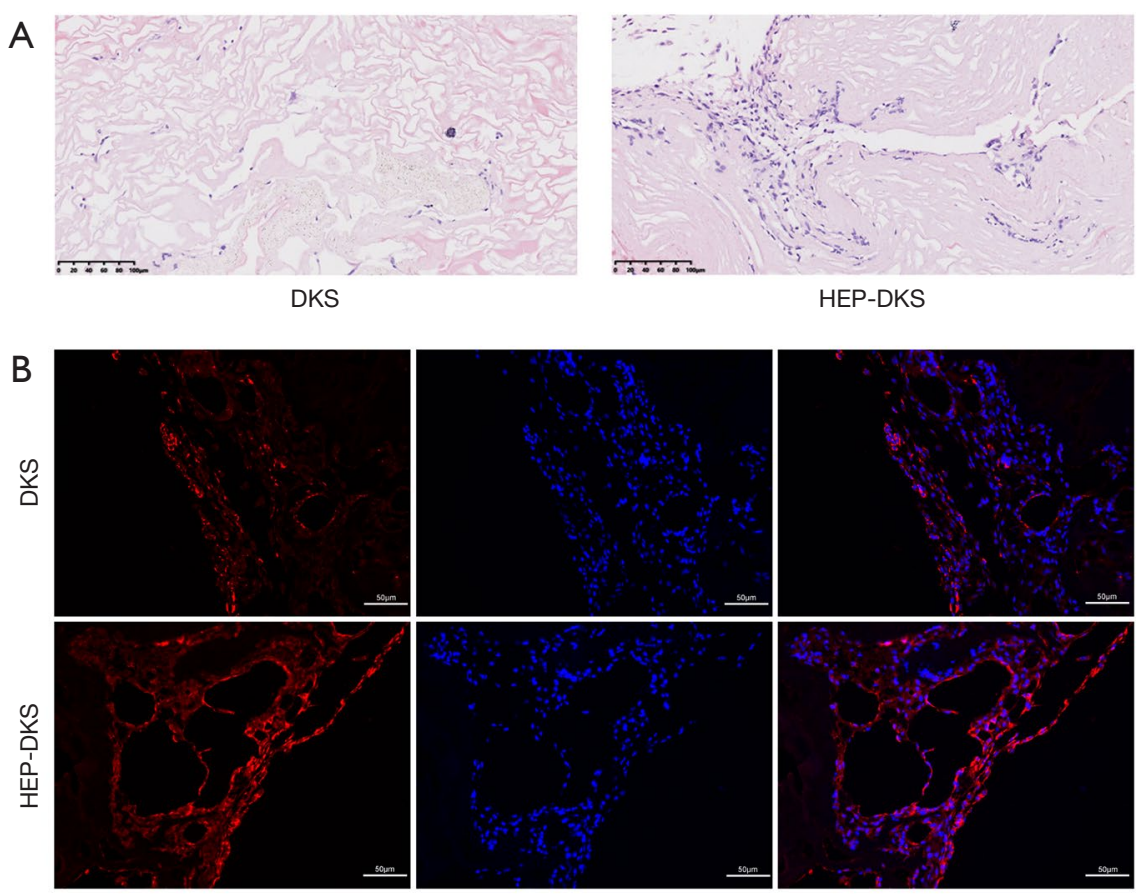

CD31

DAPI

merge

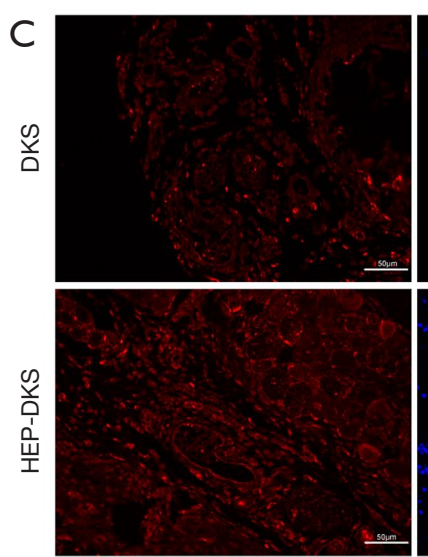

Ki67
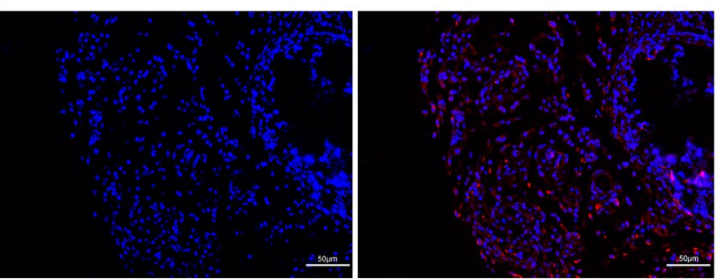

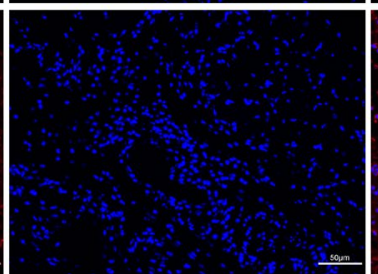

DAPI

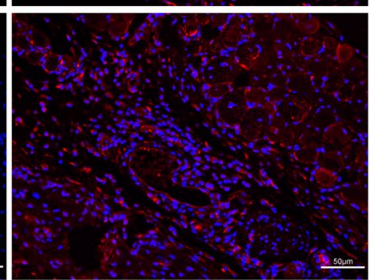

merge

Figure 5 Endothelial cells seeded into the native and heparin-modified DKS for 7 days. (A) H\&E staining of the re-endothelialized DKS showed that more HUVECs adhered around the vascular lumen in the HEP-DKS compared with the native DKS (200x). (B,C) Immunofluorescence staining of CD31 and Ki67 was conducted to assess HUVEC proliferation after cell seeding into the DKS and HEPDKS (400x). DKS, decellularized kidney scaffold; H\&E, hematoxylin and eosin; HEP-DKS, heparinized decellularized kidney scaffold; HUVECs, human umbilical vein endothelial cells.

emerged in in vivo experiments on acellular kidney stents, including thrombosis in the vasculature of the DKS and inflammatory responses of the transplanted host. In order to reduce the host inflammatory response, researchers have tried to optimize the decellularization scaffold from at least three aspects: first, by improving the decellularization process, to reduce the residual cells and antigen components of the scaffold. Second, by modifying certain active ingredients on the decellularized scaffold, to increase the compatibility of the decellularized scaffold and the host tissue. Third, by modifying anti-inflammatory drugs on the decellularized scaffold, to counteract the host inflammatory 

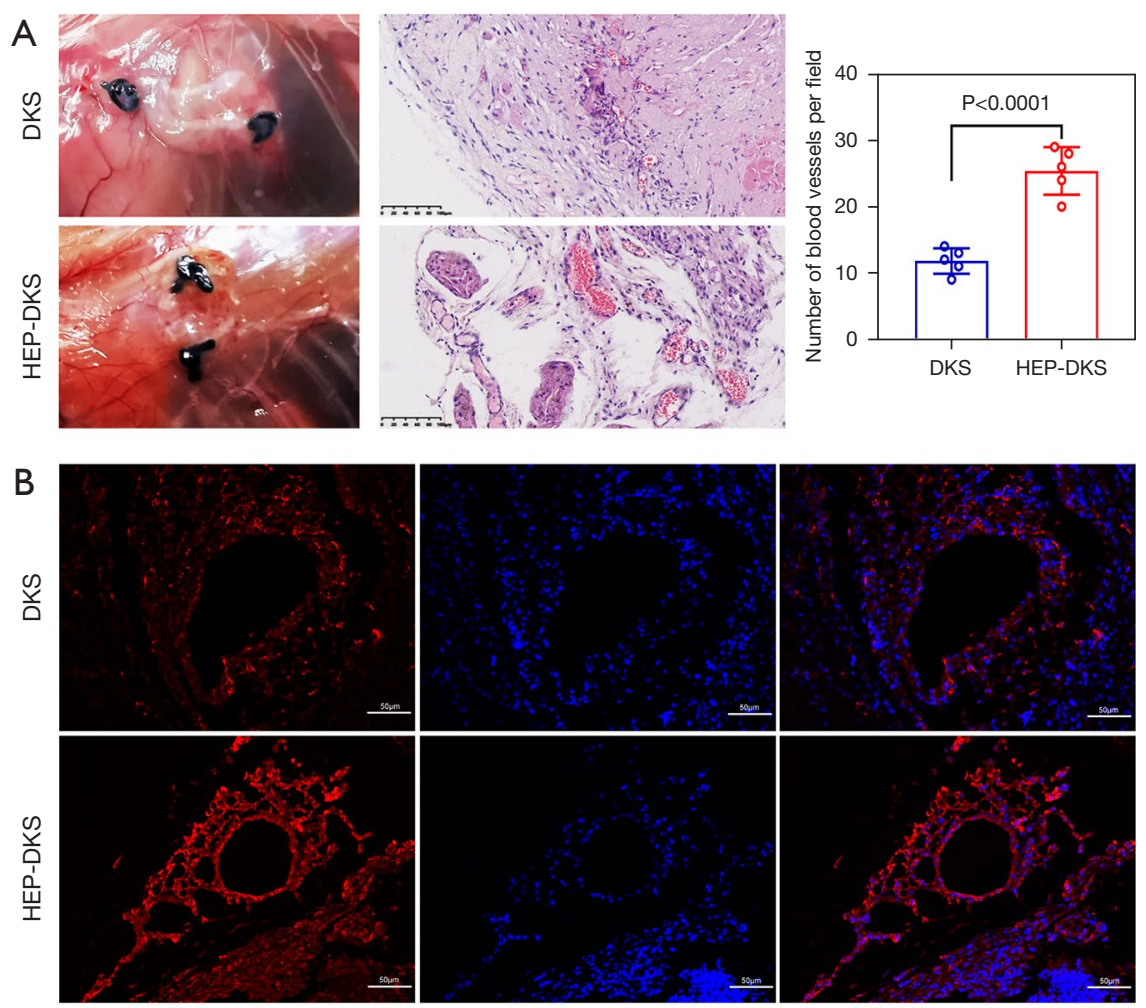

CD31

DAPI

merge

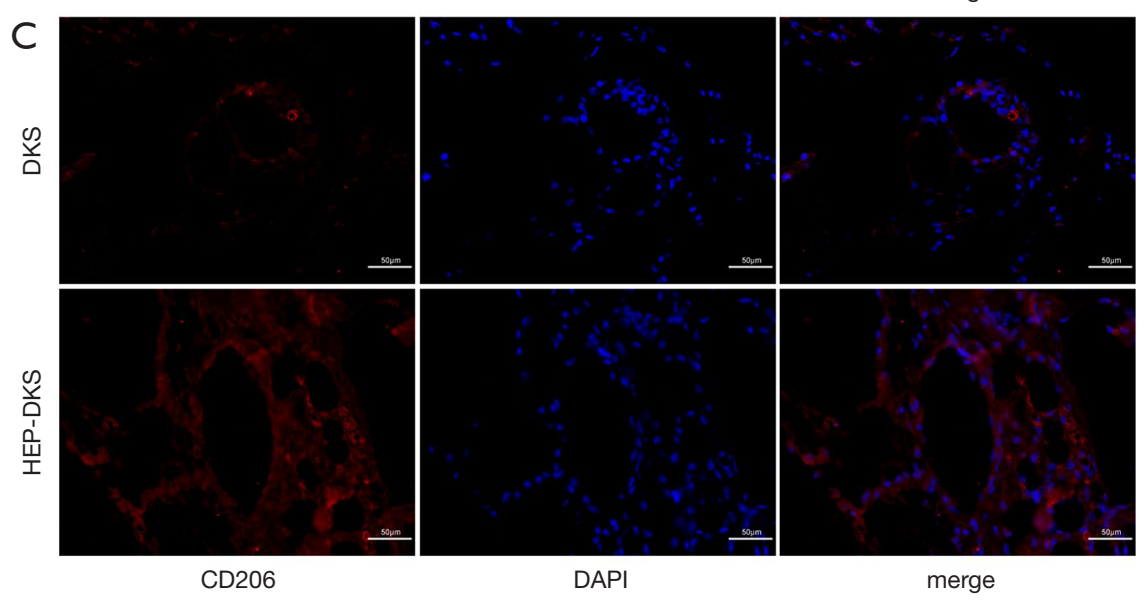

Figure 6 Angiogenesis and host inflammatory response towards the DKS in vivo. (A) Left: gross appearances of implants 3 weeks postimplantation. Both of the re-endothelialized scaffolds were biocompatible. Middle: H\&E staining showed more blood vessels in the HEPDKS group on day 21. Right: blood vessel densities of the 2 groups $(\mathrm{P}<0.0001)(200 \times)$. (B) IF staining of CD31 in the nude mouse model for the native DKS and HEP-DKS on day 21. (C) IF staining of CD206 in the nude mouse model for the native DKS and HEP-DKS on day 21 after implantation (400×). DKS, decellularized kidney scaffold; H\&E, hematoxylin and eosin; HEP-DKS, heparinized decellularized kidney scaffold; IF, immunofluorescence. 

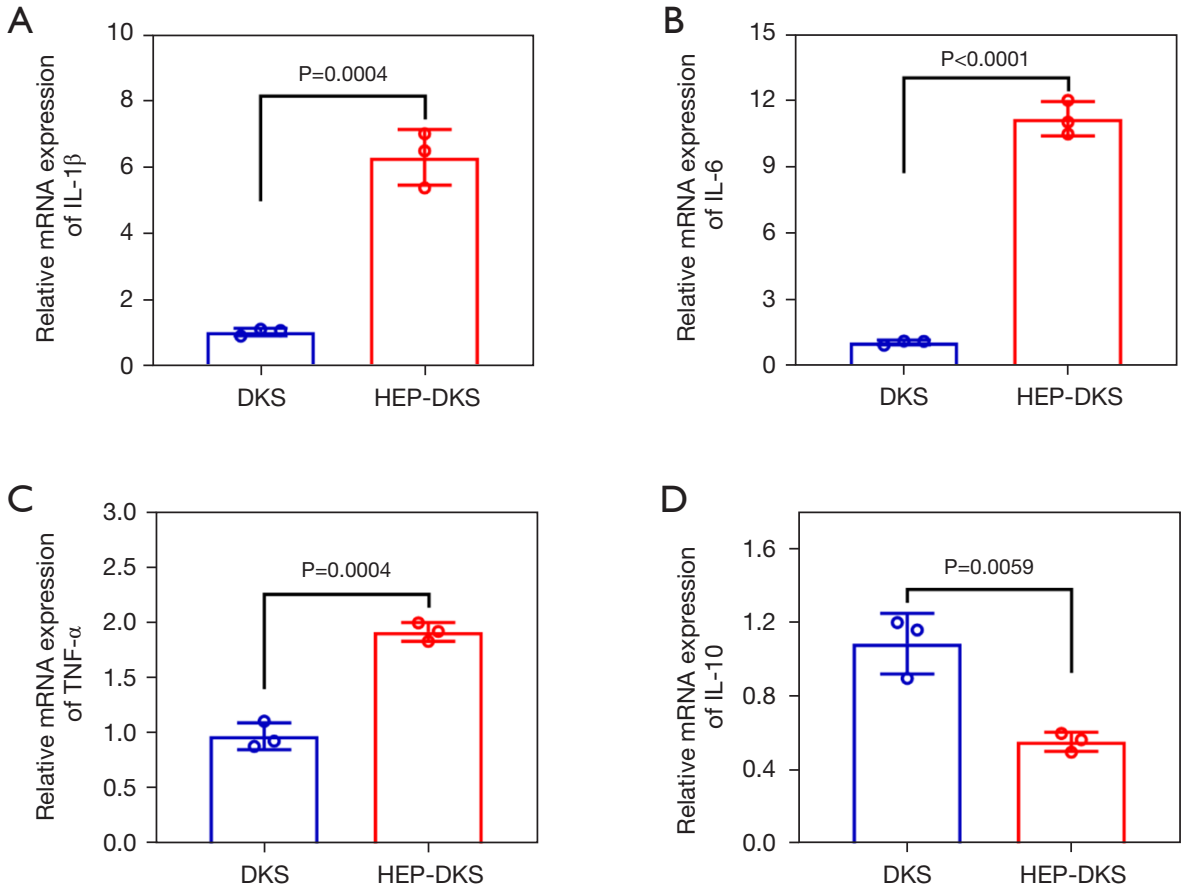

Figure 7 The expression levels of local inflammatory factors in vivo. $\mathrm{P}<0.05$. DKS, decellularized kidney scaffold; HEP-DKS, heparinized decellularized kidney scaffold.

response. Uygun et al. (15) believed that the exposed ECM in the vasculature accounted for platelet activation and thrombosis. Based on this understanding, Wan et al. (7) cross-linked GRGDSPC peptides to acellular pancreatic scaffolds, confirming that this method can help ECs adhere to the surface of blood vessels and that ECs have antiplatelet and anticoagulant effects. In another interesting study (5), an acellular pancreatic scaffold modified with platelet-rich plasma was transplanted into rats after endothelialization. It was found that the inflammatory response of the host was significantly inhibited and induced polarization dominated by M2 macrophages, which ultimately promoted tissue angiogenesis. The authors believed that platelet-rich plasma plays an important role in this research as it contains a large number of growth factors and immunoregulatory factors. In the present study, we chose heparin sodium to modify the DKS and explored its role in anti-thrombosis and antiinflammatory responses for DKS transplantation.

The method using EDC/NHS activation is a very efficient procedure for the immobilization of heparin to collagen $(4,10,16)$. In our study, collagen I, collagen IV, and fibronectin were completely retained in the process of decellularization, which was strongly demonstrated by a series of characterization experiments. This is not only critical for cell adhesion and proliferation during the re-endothelialization procedure (17), but also provides sufficient attachment points for heparin modification. TB staining and FTIR spectroscopy were performed to verify the high efficiency of the EDC/ NHS heparinization modification method. Heparin is a negatively charged linear polysaccharide consisting of 2 repeating disaccharide units (L-idonic acid, D-glucosamine, and type acetylglucosamine). Due to the unique structure of heparin, it can form a complex with antithrombin III to bind to activated coagulation blood factors and play a role in eliminating these clotting factors (18). The reaction speed of antithrombin III and thrombin is slow, but in the presence of heparin, the response rate can be increased thousands of times which can effectively inhibit the coagulation process, so the most well-known clinical effect of heparin is anticoagulation. Previous investigations have indicated that heparin has the potential to promote angiogenesis through modification into scaffolds $(16,19,20)$. Wang et al. (4) initially explored the role of heparin modification in the DKS, and they verified that the presence of heparin reduces the adhesion of platelets on the scaffold via in vitro experiments, thereby resisting thrombosis and ensuring the smooth flow of regenerated blood vessels in 
the scaffolds. In the present study, $\mathrm{H} \& \mathrm{E}$ staining and Ki67 IF staining verified the adhesion and proliferation effects of heparin on HUVECs implanted in the DKS. In addition, we carried out CD31 IF staining both in vivo and in vitro, and the results strongly demonstrated the pro-angiogenesis effect of heparin on the renal scaffolds. What makes us more confident is that we visually observed the increase of regenerated blood vessels in the renal stent modified with heparin through the naked eye and H\&E staining in vivo. We also believe that the anticoagulant effect of heparin plays an integral role in this process.

Although the decellularized scaffolds achieve minimal immunogenic residues, the host inflammatory response will inevitably have an effect on the re-endothelialization and vascular regeneration of the scaffolds, which has not yet been fully elucidated in previous reports $(4,16,17)$. Macrophages are a very heterogeneous cell population that exhibit unique phenotypes and functions in the complex microenvironment of the body. Mantovani et al. (21) believe that macrophages have a series of continuous functional states, and M1 and M2 macrophages are the 2 extremes of this continuous state. M1 type macrophages participate in the positive immune response and immune surveillance by secreting pro-inflammatory cytokines or chemokines and specifically presenting antigens M2 type macrophages have only weak antigen-presenting ability, and they can down-regulate the immune response by secreting inhibitory cytokines such as IL-10 and/or TGF- $\beta$, among others, thus playing an important role in immune regulation (22). In this study, we aimed to reveal the changes in the polarity of macrophages caused by kidney scaffolds implanted subcutaneously in nude mice after a period of growth. CD206 is a relatively specific molecular marker for M2 macrophages. We detected the changes in the expression level of CD206 by IF staining, and the results confirmed that heparin modification promoted the polarization of M1 type macrophages to M2 type in the transplanted host. We further detected the changes of IL$1 \beta$, IL-6, IL-10, and TNF- $\alpha$ expression at the mRNA level and clarified the regulatory effect of heparin modification on the inflammatory response of the host. In addition to the anticoagulant effect of heparin, there is also evidence suggesting that it has a direct anti-inflammatory effect, which greatly expands the clinical indications of heparin, including autoimmune diseases, malignant tumors, and diffuse intravascular coagulation (23). There are many explanations for the mechanism by which heparin exerts a direct anti-inflammatory effect, but there is as yet no consensus. One explanation is that heparin binds and inhibits chemokines, as well as complement, growth, and angiogenesis factors (24), and another is that heparin can bind to adhesion mediators expressed during inflammation, including selectins, integrins, and their receptors (25). There are certain limitations in our research. We only paid attention to the influence of heparin on the host environment after the DKS was implanted into animals, which proved the anti-inflammatory effect of heparin, but the specific mechanism that initiates the immune response needs to be further verified, which is our next research plan.

\section{Conclusions}

In summary, a DKS with maximum microstructure and minimum immunogenicity was prepared by arterial infusion. With the help of the EDC/NHS activation method, heparin was efficiently modified to the collagen of the scaffolds. HUVECs could attach to the tubular structure of the scaffolds and initiate re-endothelialization. In vivo and in vitro experiments demonstrated that heparin can promote the re-endothelialization and vascular regeneration of the DKS through anticoagulation. The anti-inflammatory effect of heparin on the transplanted host was initially confirmed, and it is considered that this effect may play a nonnegligible role in promoting DKS re-endothelialization and angiogenesis. The specific mechanism needs to be further clarified.

\section{Acknowledgments}

Funding: The current study was supported by the National Natural Science Foundation of China (grant No. 32070646).

\section{Footnote}

Reporting Checklist: The authors have completed the ARRIVE reporting checklist. Available at https://dx.doi. org/10.21037/tau-21-703

Data Sharing Statement: Available at https://dx.doi. org/10.21037/tau-21-703

Conflicts of Interest: All authors have completed the ICMJE uniform disclosure form (available at https://dx.doi. org/10.21037/tau-21-703). The authors have no conflicts of interest to declare. 
Ethical Statement: The authors are accountable for all aspects of the work in ensuring that questions related to the accuracy or integrity of any part of the work are appropriately investigated and resolved. The study was approved by the Ethics Committee of Shanghai Tenth People's Hospital (No. SHDSYY-2021-3647), in compliance with national guidelines for the care and use of animals.

Open Access Statement: This is an Open Access article distributed in accordance with the Creative Commons Attribution-NonCommercial-NoDerivs 4.0 International License (CC BY-NC-ND 4.0), which permits the noncommercial replication and distribution of the article with the strict proviso that no changes or edits are made and the original work is properly cited (including links to both the formal publication through the relevant DOI and the license). See: https://creativecommons.org/licenses/by-nc-nd/4.0/.

\section{References}

1. Orlando G, Farney AC, Iskandar SS, et al. Production and implantation of renal extracellular matrix scaffolds from porcine kidneys as a platform for renal bioengineering investigations. Ann Surg 2012;256:363-70.

2. Ross EA, Williams MJ, Hamazaki T, et al. Embryonic stem cells proliferate and differentiate when seeded into kidney scaffolds. J Am Soc Nephrol 2009;20:2338-47.

3. Nakayama KH, Batchelder CA, Lee CI, et al. Decellularized rhesus monkey kidney as a threedimensional scaffold for renal tissue engineering. Tissue Eng Part A 2010;16:2207-16.

4. Wang M, Bao L, Qiu X, et al. Immobilization of heparin on decellularized kidney scaffold to construct microenvironment for antithrombosis and inducing reendothelialization. Sci China Life Sci 2018;61:1168-77.

5. Zhang L, Qiu H, Wang D, et al. Enhanced vascularization and biocompatibility of rat pancreatic decellularized scaffolds loaded with platelet-rich plasma. J Biomater Appl 2020;35:313-30.

6. Sant S, Wang D, Abidi M, et al. Mechanical characterization of native and sugar-modified decellularized kidneys. J Mech Behav Biomed Mater 2021;114:104220.

7. Wan J, Wang L, Huang Y, et al. Using GRGDSPC peptides to improve re-endothelialization of decellularized pancreatic scaffolds. Artif Organs 2020;44:E172-80.

8. Ko IK, Peng L, Peloso A, et al. Bioengineered transplantable porcine livers with re-endothelialized vasculature. Biomaterials 2015;40:72-9.
9. Dai J, Qiao W, Shi J, et al. Modifying decellularized aortic valve scaffolds with stromal cell-derived factor1alpha loaded proteolytically degradable hydrogel for recellularization and remodeling. Acta Biomater 2019;88:280-92.

10. Wissink MJ, Beernink R, Pieper JS, et al. Immobilization of heparin to EDC/NHS-crosslinked collagen. Characterization and in vitro evaluation. Biomaterials 2001;22:151-63.

11. Foreman KJ, Marquez N, Dolgert A, et al. Forecasting life expectancy, years of life lost, and all-cause and causespecific mortality for 250 causes of death: reference and alternative scenarios for 2016-40 for 195 countries and territories. Lancet 2018;392:2052-90.

12. Sobreiro-Almeida R, Quinteira R, et al. Renal Regeneration: The Role of Extracellular Matrix and Current ECM-Based Tissue Engineered Strategies. Adv Healthc Mater 2021;10:e2100160.

13. Hussein KH, Saleh T, Ahmed E, et al. Biocompatibility and hemocompatibility of efficiently decellularized whole porcine kidney for tissue engineering. J Biomed Mater Res A 2018;106:2034-47.

14. Brown B, Lindberg K, Reing J, et al. The basement membrane component of biologic scaffolds derived from extracellular matrix. Tissue Eng 2006;12:519-26.

15. Uygun BE, Soto-Gutierrez A, Yagi H, et al. Organ reengineering through development of a transplantable recellularized liver graft using decellularized liver matrix. Nat Med 2010;16:814-20.

16. Xu L, Guo Y, Huang Y, et al. Constructing heparinmodified pancreatic decellularized scaffold to improve its re-endothelialization. J Biomater Appl 2018;32:1063-70.

17. Zhou L, Wang Z, Wang Z, et al. Effect of heparinization on promoting angiogenesis of decellularized kidney scaffolds. J Biomed Mater Res A 2021;109:1979-89.

18. Hemker HC. A century of heparin: past, present and future. J Thromb Haemost 2016;14:2329-38.

19. Jiang B, Suen R, Wertheim JA, et al. Targeting Heparin to Collagen within Extracellular Matrix Significantly Reduces Thrombogenicity and Improves Endothelialization of Decellularized Tissues. Biomacromolecules 2016;17:3940-8.

20. Hussein KH, Park KM, Kang KS, et al. Heparin-gelatin mixture improves vascular reconstruction efficiency and hepatic function in bioengineered livers. Acta Biomater 2016;38:82-93.

21. Mantovani A, Sica A, Sozzani S, et al. The chemokine system in diverse forms of macrophage activation and 
polarization. Trends Immunol 2004;25:677-86.

22. Van Ginderachter JA, Movahedi K, Hassanzadeh Ghassabeh G, et al. Classical and alternative activation of mononuclear phagocytes: picking the best of both worlds for tumor promotion. Immunobiology 2006;211:487-501.

23. Poterucha TJ, Libby P, Goldhaber SZ. More than an anticoagulant: Do heparins have direct anti-inflammatory effects? Thromb Haemost 2017;117:437-44.

24. Chiodelli P, Bugatti A, Urbinati C, et al. Heparin/
Heparan sulfate proteoglycans glycomic interactome in angiogenesis: biological implications and therapeutical use. Molecules 2015;20:6342-88.

25. Fritzsche J, Alban S, Ludwig RJ, et al. The influence of various structural parameters of semisynthetic sulfated polysaccharides on the $\mathrm{P}$-selectin inhibitory capacity. Biochem Pharmacol 2006;72:474-85.

(English Language Editor: C. Betlzar)

Cite this article as: Xie J, Wan J, Tang X, Li W, Peng B. Heparin modification improves the re-endothelialization and angiogenesis of decellularized kidney scaffolds through antithrombosis and anti-inflammation in vivo. Transl Androl Urol 2021;10(9):3656-3668. doi: 10.21037/tau-21-703 